\title{
A leptin-regulated circuit controls glucose mobilization during noxious stimuli
}

\author{
Jonathan N. Flak,, Deanna Arble, ${ }^{2}$ Warren Pan, ${ }^{1,3}$ Christa Patterson, ${ }^{1}$ Thomas Lanigan, ${ }^{1}$ Paulette B. Goforth, ${ }^{4}$ Jamie Sacksner, ${ }^{1}$ \\ Maja Joosten, ${ }^{5}$ Donald A. Morgan, ${ }^{6}$ Margaret B. Allison,${ }^{17}$ John Hayes, ${ }^{8}$ Eva Feldman, ${ }^{8}$ Randy J. Seeley, ${ }^{2}$ David P. Olson, ${ }^{9}$ \\ Kamal Rahmouni, ${ }^{6}$ and Martin C. Myers Jr. ${ }^{1,2,7}$ \\ DDepartment of Internal Medicine, ${ }^{2}$ Department of Surgery, ${ }^{3}$ Graduate Program in Cellular and Molecular Biology, and ${ }^{4}$ Department of Pharmacology, University of Michigan, Ann Arbor, Michigan, USA. \\ ${ }^{5}$ Radboud University, Nijmegen, Netherlands. ${ }^{6}$ Department of Pharmacology, University of lowa, lowa City, lowa, USA. Department of Molecular and Integrative Physiology, \\ ${ }^{8}$ Department of Neurology, and ${ }^{9}$ Division of Endocrinology, Department of Pediatrics and Communicable Diseases, University of Michigan, Ann Arbor, Michigan, USA.
}

\begin{abstract}
Adipocytes secrete the hormone leptin to signal the sufficiency of energy stores. Reductions in circulating leptin concentrations reflect a negative energy balance, which augments sympathetic nervous system (SNS) activation in response to metabolically demanding emergencies. This process ensures adequate glucose mobilization despite low energy stores. We report that leptin receptor-expressing neurons (LepRb neurons) in the periaqueductal gray (PAC), the largest population of LepRb neurons in the brain stem, mediate this process. Application of noxious stimuli, which often signal the need to mobilize glucose to support an appropriate response, activated PAC LepRb neurons, which project to and activate parabrachial nucleus (PBN) neurons that control SNS activation and glucose mobilization. Furthermore, activating PAC LepRb neurons increased SNS activity and blood glucose concentrations, while ablating LepRb in PAG neurons augmented glucose mobilization in response to noxious stimuli. Thus, decreased leptin action on PAC LepRb neurons augments the autonomic response to noxious stimuli, ensuring sufficient glucose mobilization during periods of acute demand in the face of diminished energy stores.
\end{abstract}

\section{Introduction}

The hormone leptin, which is produced by adipocytes to signal the repletion of body energy (fat) stores, acts via the leptin receptor (LepRb) on specialized populations of brain neurons to control food intake, energy expenditure, and other metabolic parameters (1-4). The fall in circulating leptin concentrations during negative energy balance increases hunger and initiates an energy-sparing neuroendocrine program characterized by decreased sympathetic nervous system (SNS) tone and diminished activity of the thyroid, reproductive, and growth axes, among others. This response not only tends to replenish body energy stores by increasing feeding relative to energy utilization when food is available, but also preserves remaining energy stores in the face of continued famine, increasing the chances of survival until food becomes available.

The withdrawal of leptin action from LepRb neurons in the hypothalamus mediates most of these orexigenic and energysparing responses to negative energy balance (5). While brain stem sites contain many LepRb neurons, roles for most populations of brain stem LepRb neurons, including the largest population (located in the periaqueductal gray [PAG]), remain undefined $(6,7)$.

In isolation, the energy-sparing neuroendocrine response to negative energy balance carries a potential risk, however. The reduction of energy stores coupled with decreased SNS tone impairs the ability to respond to acute, potentially life-threatening

Conflict of interest: The authors have declared that no conflict of interest exists Submitted: August 15, 2016; Accepted: June 2, 2017

Reference information: J Clin Invest. 2017;127(8):3103-3113.

https://doi.org/10.1172/JCI90147. emergencies that require the robust mobilization of metabolic fuels, such as glucose. Thus, during negative energy balance, meeting acutely elevated metabolic needs requires a system that increases the drive to mobilize glucose beyond that which is required when energy reserves are replete. Furthermore, this system must activate specifically in response to metabolic emergencies without otherwise altering SNS tone and glucose mobilization (otherwise, this would blunt the energy-sparing response to negative energy balance). Indeed, we previously defined a system that augments the counterregulatory response (CRR) to hypoglycemia when energy stores are diminished: hypoglycemia activates cholecystokinin-containing (CCK-containing) neurons in the hindbrain parabrachial nucleus (PBN), which project to the ventromedial hypothalamic nucleus (VMN) (which plays a crucial role in the CRR) $(8,9)$. Some PBN CCK neurons contain LepRb; during negative energy balance, the withdrawal of leptin from these PBN Lep$\mathrm{Rb}$ cells augments their response to low glucose, enhancing glucose mobilization during hypoglycemia without altering baseline endocrine function, energy balance, or glucose homeostasis (8).

We reasoned that other stimuli that signal the need for robust SNS activation and glucose mobilization might similarly engage the $\mathrm{PBN} \rightarrow \mathrm{VMN}$ circuit to meet acutely increased metabolic demands when energy stores are low. Because noxious stimuli (such as pain) increase blood glucose and often indicate the need for SNS activation and nutrient mobilization, we sought to understand the potential role for hindbrain LepRb neurons in modulating the response to noxious stimuli in line with the repletion state of body energy stores. We find that LepRb neurons in the PAG (which plays crucial roles in responding to noxious stimuli; refs. 10-13) lie 
A

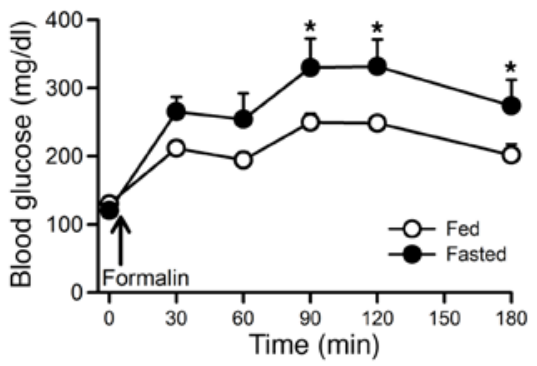

Formalin
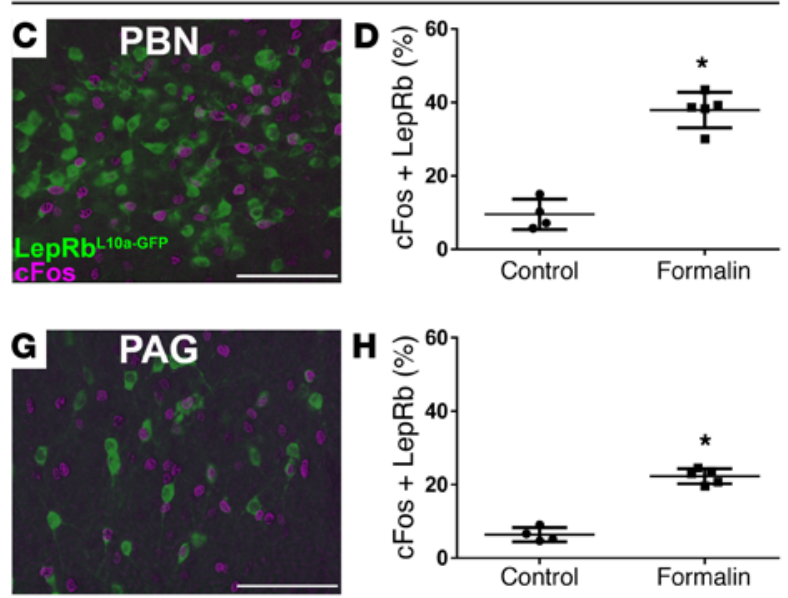

B

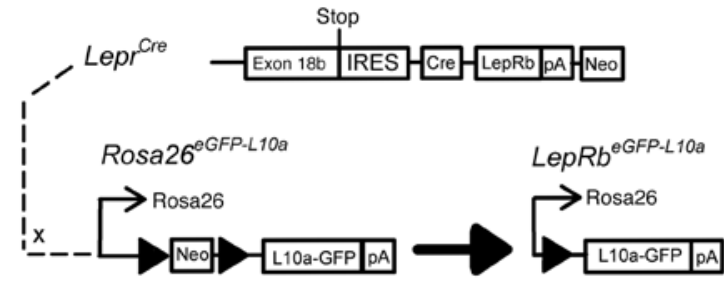

2dg
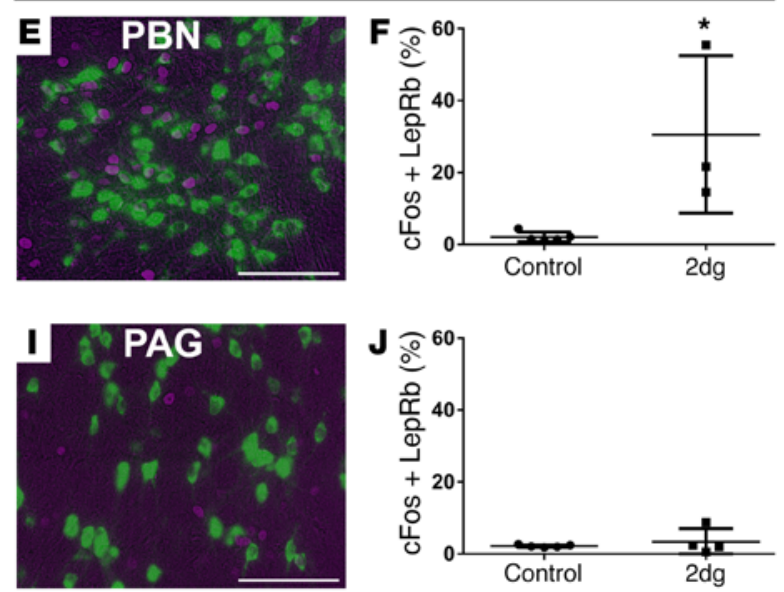

Figure 1. Hyperglycemic and c-Fos responses to noxious stimuli. (A) Ad libitum-fed $(n=10)$ and overnight-fasted $(n=8)$ C57BL/6 mice were injected with intra-hind paw formalin $(5 \%, 20 \mu \mathrm{l})$, and blood glucose concentrations were measured over the subsequent 3 hours. Data are plotted as mean \pm SEM. (B) LepRb ${ }^{\text {eCFP-L10a }}$ mice were treated with $(\mathbf{C}, \mathbf{D}, \mathbf{G}, \mathbf{H})$ intra-hind paw formalin $(n=4$, veh; $n=5$, formalin) as in $\mathbf{A}$ or $(\mathbf{E}, \mathbf{F}, \mathbf{I}, \mathbf{J}) 2 \mathrm{DG}(n=5$, veh; $n=4,2 \mathrm{DG})(500$ $\mathrm{mg} / \mathrm{kg}$, i.p.) and perfused under anesthesia. Brains were collected and sectioned; sections were stained for c-Fos (DAB, purple) and GFP (green). Images (C, E, G, I) are representative of 4-5 similar animals for each treatment. Graphs show colocalized cells/total LepRb cells, plotted as percentage, mean \pm SEM. ${ }^{*} P<0.05$. Scale bars: $100 \mu \mathrm{m}$. Data in panel $\mathbf{A}$ were analyzed by 2-way repeated-measures ANOVA with Fisher's LSD post hoc test, and data in panels $\mathbf{D}, \mathbf{F}$, $\mathbf{H}$, and $\mathbf{J}$ were analyzed by 1 -tailed $t$ test.

upstream of the $\mathrm{PBN} \rightarrow \mathrm{VMN}$ circuit. Diminished leptin action on these LepRb ${ }^{\text {PAG }}$ neurons augments the hyperglycemic response to noxious stimuli, ensuring appropriately elevated autonomic output to mediate sufficient glucose mobilization in response to acute metabolic need under conditions of negative energy balance.

\section{Results}

Noxious stimuli activate LepRb neurons in the PBN and PAG. Pain and other noxious stimuli activate the SNS and promote the mobilization of metabolic fuels to support the ability to cope with the stimulus-evoking event. Thus, short-term survival when faced with a noxious stimulus during negative energy balance requires a brain system that can overcome low SNS tone and depleted energy stores to support nutrient mobilization. Indeed, a 12-hour fast enhances the hyperglycemic response to the injection of $5 \%$ formalin into the footpad of the hind paw (a common noxious stimulus model; refs. 14-16) (Figure 1A).

Since the withdrawal of leptin from leptin-inhibited LepRb neurons in the PBN (LepRb ${ }^{\mathrm{PBN}}$ cells) enhances SNS activation and glucose mobilization during hypoglycemia (8), mitigating the impairment of the CRR that would otherwise occur during negative energy balance, we sought to determine whether the LepRb ${ }^{\text {PBN }}$ circuitry might similarly modulate the response to noxious stimuli. We thus examined the potential activation of LepRb $\mathrm{B}^{\mathrm{PBN}}$ cells by noxious stimuli, using the criterion of immunohistochemically detectable c-Fos induction in Lepr ${ }^{\text {Cre }}$ Rosa26 $6^{\text {L10a-GFP }}$ (LepRb ${ }^{\mathrm{L} 10 \mathrm{a}-\mathrm{GFP}}$; ref. 17) mice (Figure 1B), which express an L10a-GFP fusion protein in LepRb neurons, enabling their detection by $\alpha$ GFP immunohistochemistry (Figure 1 and Supplemental Figure 1; supplemental material available online with this article; https://doi.org/ 10.1172/JCI90147DS1). Indeed, in LepRb ${ }^{\mathrm{L10a}-\mathrm{GFP}}$ mice, formalin injection into the footpad promoted c-Fos accumulation in $38 \%$ of LepRb ${ }^{\mathrm{PBN}}$ neurons (562 \pm 23 cells, mean \pm SEM; $n=5$ ) (Figure $1, \mathrm{C}$ and D), similar to the effect of glucoprivation by the injection of 2-deoxyglucose (2DG) (which mimics hypoglycemia at the cellular level) on these cells ( $568 \pm 6$ cells, mean \pm SEM; $n=3$ ) (Figure 1, E and F), suggesting that LepRb ${ }^{\mathrm{PBN}}$ neurons might play a role in modulating the response to noxious stimuli, in addition to hypoglycemia, during negative energy balance. Additionally, formalin injection activated approximately $23 \%$ of the LepRb neurons $(1862 \pm 106$ cells, mean $\pm \mathrm{SEM} ; n=5)$ in the neighboring PAG (LepRb ${ }^{\text {PAG }}$ neurons) (Figure $1, \mathrm{G}$ and $\mathrm{H}$ ), although glucoprivation did not activate LepRb ${ }^{\text {PAG }}$ cells (Figure 1 , I and J).

Integration of $L e p R b^{P A G}$ neurons with the $P B N \rightarrow V M N$ circuit. Because noxious stimulus exposure activates both LepRb ${ }^{\mathrm{PAG}}$ and LepRb ${ }^{\text {PBN }}$ neurons (while glucoprivation activates only LepRb ${ }^{\text {PBN }}$ cells), and since the PAG receives and integrates signals related to noxious stimuli, we hypothesized that LepRb ${ }^{\text {PAG }}$ neurons might 
A AAV-Syn-GFP
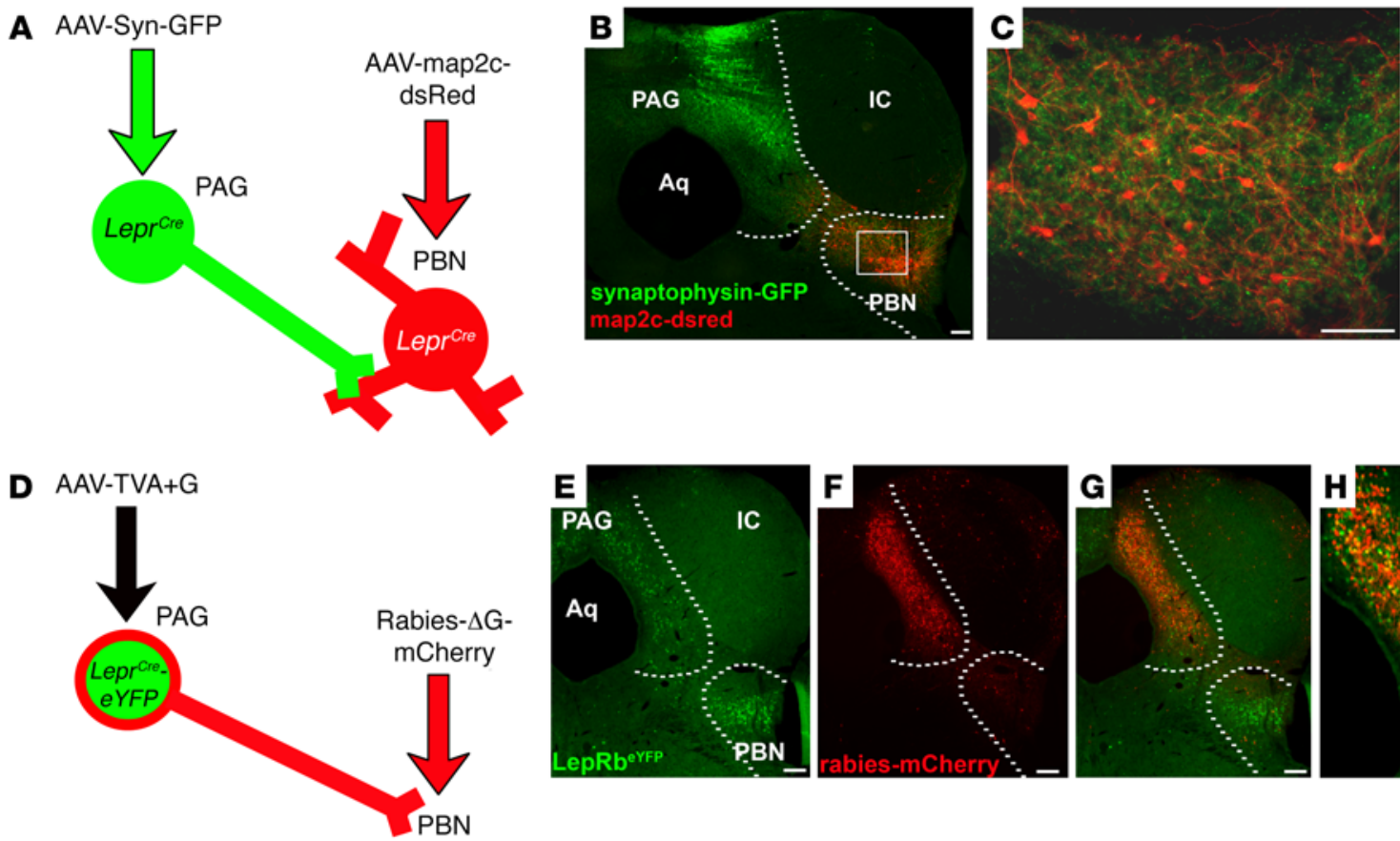
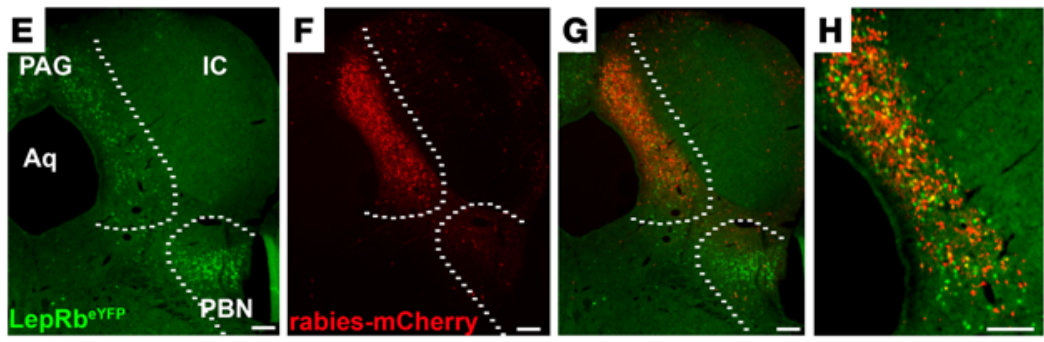

Figure 2. PAG ${ }^{\text {LepRb }}$ neurons project to the PBN. (A) Lepr ${ }^{\text {Cre }}$ mice were injected with AAV-Syn-GFP into the PAG and AAV-map2c-dsRed into the PBN. The mice were perfused; (B and C) brains were collected, sectioned, and stained for GFP (green) and dsRed (red). Images are representative of 3 similar cases. Dashed lines indicate the boundaries of the PAG and PBN. Box in B shows the zoomed-in area represented in C. (D) LeprCre/eYFP mice were injected with AAV-TVA+G into the PAG; 2 weeks later, defective pseudotyped mCherry-expressing rabies virus (Rabies- $\Delta \mathrm{G}$-mCherry) was injected into the PBN. Five days later, mice were perfused and brains were collected and sectioned. Brain sections were stained for GFP (green) and dsRed (red) (E and F). Images are representative of 4 similar cases. $\mathbf{H}$ shows an enlarged image of G. Scale bars: $100 \mu \mathrm{m}$. IC, inferior colliculus; Aq, cerebral aqueduct.

represent the means by which information regarding noxious stimuli enters the $\mathrm{PBN} \rightarrow \mathrm{VMN}$ circuit. To test this, we injected AAV-SynGFP (which mediates the Cre-inducible expression of a synaptophysin-GFP fusion protein to reveal synaptic terminals) into the PAG of Lepr ${ }^{\text {Cre }}$ mice; at the same time, we injected AAVmap2c-dsRed (which mediates the Cre-inducible expression of a map2c-dsRed fusion protein that identifies the dendritic tree) into the PBN of the same mice (Figure 2A). This revealed the projection field of LepRb ${ }^{\mathrm{PAG}}$ neurons, which densely innervate the $\mathrm{PBN}$, and demonstrated that many of the synaptic terminals from these cells lie in the $\mathrm{PBN}$ region that contains the dendritic tree of LepRb ${ }^{\text {PBN }}$ neurons (Figure 2, B and C). We also found terminals from LepRb ${ }^{\mathrm{PAG}}$ neurons in the dorsomedial nucleus of the hypothalamus (DMH) and dorsomedial thalamus (Supplemental Figure 2), although these were much sparser than those in the PBN.

We employed rabies-mediated monosynaptic retrograde tracing (18) to examine the circuitry of PBN-projecting LepR $\mathrm{B}^{\mathrm{PAG}}$ cells. In this system, the injection of the AAV-TVA+G helper virus mediates expression of TVA in Cre-containing neurons to permit their infection by a pseudotyped, G-deleted, mCherry-expressing rabies virus (Rabies- $\Delta$ G-mCherry). AAV-TVA+G also mediates the Credependent expression of $G$, enabling the passage of Rabies- $\Delta G$ mCherry one synapse retrograde to the Cre-expressing neuron. We injected AAV-TVA+G into the PAG of Lepr ${ }^{\text {Cre-eYFP }}$ mice and then delivered Rabies- $\Delta$ G-mCherry into the PBN (Figure 2, D-H). This not only infected many LepRb ${ }^{\mathrm{PAG}}$ cells with Rabies- $\Delta \mathrm{G}-\mathrm{mCherry}$ (consistent with our identification of the PBN as a major projection target of LepRb ${ }^{\text {PAG }}$ cells), but also revealed numerous Rabies- $\Delta \mathrm{G}$ -
mCherry-containing local afferents of PBN-projecting LepRb ${ }^{\text {PAG }}$ cells, especially in the PAG. We also observed afferents to the LepRb ${ }^{\text {PAG }} \rightarrow$ PBN circuit in more distant sites, including the dorsal raphe (DR) (Supplemental Figure 3); we observed few afferents elsewhere and none in the spinal cord, suggesting little or no direct input from peripheral nociceptive neurons. Thus, most of the neural signals to the LepRb ${ }^{\mathrm{PAG}} \rightarrow \mathrm{PBN}$ circuit enter via other PAG cells.

Since LepRb ${ }^{\text {PAG }}$ cells project to the PBN and contain stimulatory neurotransmitters (such as Glu and CCK), activation of LepRb ${ }^{\text {PAG }}$ cells should stimulate neurons in the PBN. To test this, we utilized AAV-hMD3q, which mediates the Cre-dependent expression of the Gq-coupled designer receptor exclusively acti-

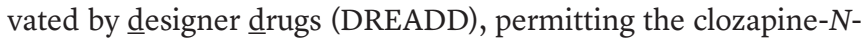
oxide-stimulated (CNO-stimulated) activation of Cre-expressing neurons in the region where the virus is injected. We injected AAV-hMD3q separately into the PAG or PBN of Lepr ${ }^{\text {Cre }}$ mice and examined the induction of c-Fos in these regions following the administration of CNO (Supplemental Figure 4). CNO treatment of PAG-injected mice promoted the accumulation of c-Fos in the PAG and PBN, while CNO promoted robust c-Fos accumulation in the PBN of PBN-injected mice, but little in the PAG. Thus, LepRb ${ }^{\text {PAG }}$ neurons lie afferent to and stimulate PBN neurons.

To determine whether PAG neurons, including LepR $\mathrm{B}^{\mathrm{PAG}}$ cells, lie in direct synaptic contact with the LepRb ${ }^{\mathrm{PBN}} \rightarrow \mathrm{VMN}$ circuit, we injected AAV-TVA $+G$ into the PBN of LepRb ${ }^{\mathrm{L} 10 \mathrm{a}-\mathrm{GFP}}$ mice, followed by the injection of Rabies- $\Delta \mathrm{G}$-mCherry into the VMN to mediate the infection of VMN-projecting LepRb ${ }^{\mathrm{PBN}}\left(\mathrm{LepRb}^{\mathrm{PBN}} \rightarrow \mathrm{VMN}\right)$ neurons specifically and to label the cells that lie 1 synapse affer- 

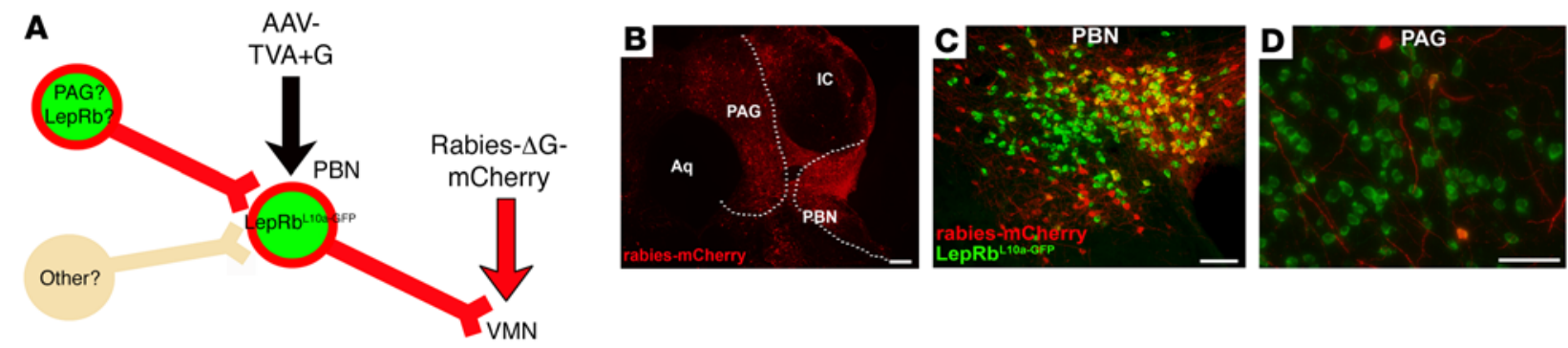

Figure 3. PAG ${ }^{\text {LepRb }}$ neurons innervate VMN-projecting PBN ${ }^{\text {LepRb }}$ neurons. (A) LepRb ${ }^{\text {eCFP-L10a }}$ mice were injected with AAV-TVA+C into the PBN, followed by defective pseudotyped mCherry-expressing rabies virus (Rabies- $\Delta G$-mCherry) injection into the VMN. Five days later, the mice were perfused, and brains were collected, sectioned, and stained for GFP (green) and dsRed (red) (B-D). Shown are images representative of 4 similar cases: (B) PAG, PBN, and surrounding areas (mCherry only); (C) PBN (merged image); (D) PAG (merged image). Dashed lines in B denote the boundaries of the PAG and PBN. Scale bars: $100 \mu \mathrm{m}$.

ent to these cells (Figure 3A). This analysis revealed Rabies- $\Delta \mathrm{G}-$ mCherry-infected cells in the PBN as well as identifying afferents of LepR $\mathrm{B}^{\mathrm{PBN}} \rightarrow \mathrm{VMN}$ neurons in the PAG and in several other nearby areas (Figure 3B). We also observed afferents of $\mathrm{LepRb} b^{\mathrm{PBN}} \rightarrow \mathrm{VMN}$ neurons in more distal regions, including the DR, the paraventricular nucleus of the hypothalamus, the bed nucleus of the stria terminalis, and the central nucleus of the amygdala (CeA) (Supplemental Figure 5). In addition to demonstrating the expected infection of many GFP-containing LepRb ${ }^{\mathrm{PBN}}$ cells by intra-VMN Rabies- $\Delta$ G-mCherry (Figure $3 C$ ), some $(<10 \%)$ of the PAG neurons afferent to the LepRb $b^{\mathrm{PBN}} \rightarrow \mathrm{VMN}$ cells contained GFP (Figure

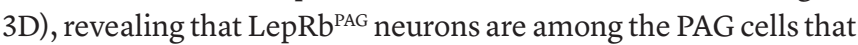
project to and synapse on VMN-projecting LepRb ${ }^{\mathrm{PBN}}$ cells.

$L e p R b^{P A G}$ neurons activate the SNS and increase blood glucose. Taken as a whole, our data suggest that LepR $b^{\mathrm{PAG}}$ cells project to and activate neurons in the $\mathrm{PBN} \rightarrow \mathrm{VMN}$ circuit, implying that activating LepRb ${ }^{\mathrm{PAG}}$ neurons should mimic the CRR, activating the SNS and increasing blood glucose concentrations. To determine the physiologic and behavioral responses to the activation of $\mathrm{LepRb}^{\mathrm{PAG}}$ and LepRb ${ }^{\mathrm{PBN}}$ cells, we injected AAV-hMD3q into the PAG or PBN of Lepr ${ }^{\text {Cre }}$ mice and examined their responses to CNO (Figure 4A). We observed $1663 \pm 364$ (mean \pm SEM; $n=7$ ) PBN hM3Dq-expressing cells and $2853 \pm 422$ (mean \pm SEM; $n=9$ ) PAG hM3Dq-expressing cells in our study. We initially observed that activation of either set of LepRb neurons increased locomotor activity (Supplemental Figure 6), consistent with increased SNS drive following the activation of these cells. Indeed, $\mathrm{CNO}$ administration to mice expressing hMD3q either in LepRb ${ }^{\mathrm{PBN}}$ or LepRb ${ }^{\mathrm{PAG}}$ cells increased adrenal sympathetic nerve activity (SNA) (Figure 4, $\mathrm{B}$ and $\mathrm{C}$ ), although the activation mediated by the LepR $\mathrm{b}^{\mathrm{PBN}}$ neurons became statistically significant at earlier times than for the $\mathrm{LepRb}^{\mathrm{PAG}}$ neurons. Furthermore, activation of $\mathrm{LepRb}^{\mathrm{PAG}}$ or LepRb ${ }^{\mathrm{PBN}}$ neurons increased blood glucose concentrations (Figure 4, D and E). Thus, LepRb ${ }^{\mathrm{PAG}}$ neurons, like LepRb ${ }^{\mathrm{PBN}}$ cells, promote SNS activity and increased blood glucose - consistent with a role for LepRb ${ }^{\mathrm{PAG}}$ neurons in controlling glucose mobilization in response to activating stimuli (e.g., noxious stimuli).

Hypercapnia normally signifies an increase in cellular respiration/metabolic demand that is not appropriately compensated by breathing; the PAG participates in the ventilatory response to hypercapnia (19-22). To determine whether LepRb ${ }^{\mathrm{PAG}}$ neurons might also participate in other autonomic responses to poten- tially noxious stimuli that reflect increased metabolic demand, we examined the ventilatory response to hypercapnia in animals expressing hM3Dq in LepR $\mathrm{B}^{\mathrm{PAG}}$ neurons (Figure $4 \mathrm{~F}$ ). CNO administration increased breathing frequency in $3 \% \mathrm{CO}_{2}$, but not in room air, consistent with augmented SNS drive to respiratory centers. Thus, in addition to increasing SNS outflow to the adrenal gland and elevating blood glucose concentrations, activation of LepRb ${ }^{\text {PAG }}$ cells augments the ventilatory response to hypercapnia, consistent with a role for these cells in promoting appropriate autonomic responses when metabolic demand is acutely elevated.

Ablation of LepRb from the PAG augments the hyperglycemic and ventilatory responses to noxious stimuli. To determine whether leptin action via brain stem LepRb neurons modulates glucose mobilization in response to noxious stimuli, we evaluated the hyperglycemic responses to noxious stimuli in LepRb ${ }^{\mathrm{CCK}} \mathrm{KO}$ mice $\left(C c R^{C r e} L_{e p r^{f / f}}\right)$, in which LepRb is ablated from CCK-expressing brain stem neurons (including LepRb ${ }^{\mathrm{PBN}}$ cells, a substantial number of LepRb ${ }^{\text {PAG }}$ neurons, and a smaller number of LepRb neurons in the Edinger-Westfall [EW] nucleus) (8) (Figure 5A). As we previously showed (8), the hyperglycemic response to glucoprivation is accentuated and prolonged in LepRb ${ }^{\mathrm{CCK}} \mathrm{KO}$ mice (Figure $5 \mathrm{~B}$ ), consistent with the role for LepR $\mathrm{R}^{\mathrm{PBN}}$ neurons in augmenting the CRR to glucoprivation when leptin action is low. Furthermore, LepR $b^{\text {cck }} \mathrm{KO}$ mice demonstrated an exaggerated hyperglycemic response to formalin injection, revealing that decreased leptin action on LepRb ${ }^{\mathrm{CCK}}$ neurons augments glucose mobilization in response to noxious stimuli (Figure 5C).

Since leptin action in Lep $\mathrm{Rb}^{\mathrm{CCK}} \mathrm{KO}$ mice is only partially interrupted in the PAG and is also ablated in the EW and PBN (8), we sought to determine the role of PAG leptin action specifically in the response to noxious stimuli. We thus injected AAV-Cre or AAV-mCherry (control) into the PAG of Lepr $r^{f / / f}$ mice, disrupting LepRb specifically in the PAG (LepRb ${ }^{\mathrm{PAG}} \mathrm{KO}$ mice) to reveal the role of LepRb ${ }^{\mathrm{PAG}}$ neurons in leptin action (Figure 6A and Supplemental Figure 7). Food intake, body weight, glucose tolerance, insulin-induced hypoglycemia, and the hyperglycemic response to glucoprivation were not altered in LepR $\mathrm{B}^{\mathrm{PAG}} \mathrm{KO}$ mice (Figure 6, $\mathrm{B}-\mathrm{F}$ ), consistent with the notion that LepRb ${ }^{\mathrm{PBN}}$ cells (rather than LepRb ${ }^{\mathrm{PAG}}$ neurons) augment the CRR to hypoglycemia/glucoprivation when leptin action is decreased.

The hyperglycemic response to formalin injection was significantly enhanced in LepRb ${ }^{\mathrm{PAG}} \mathrm{KO}$ mice, however, revealing that 
A
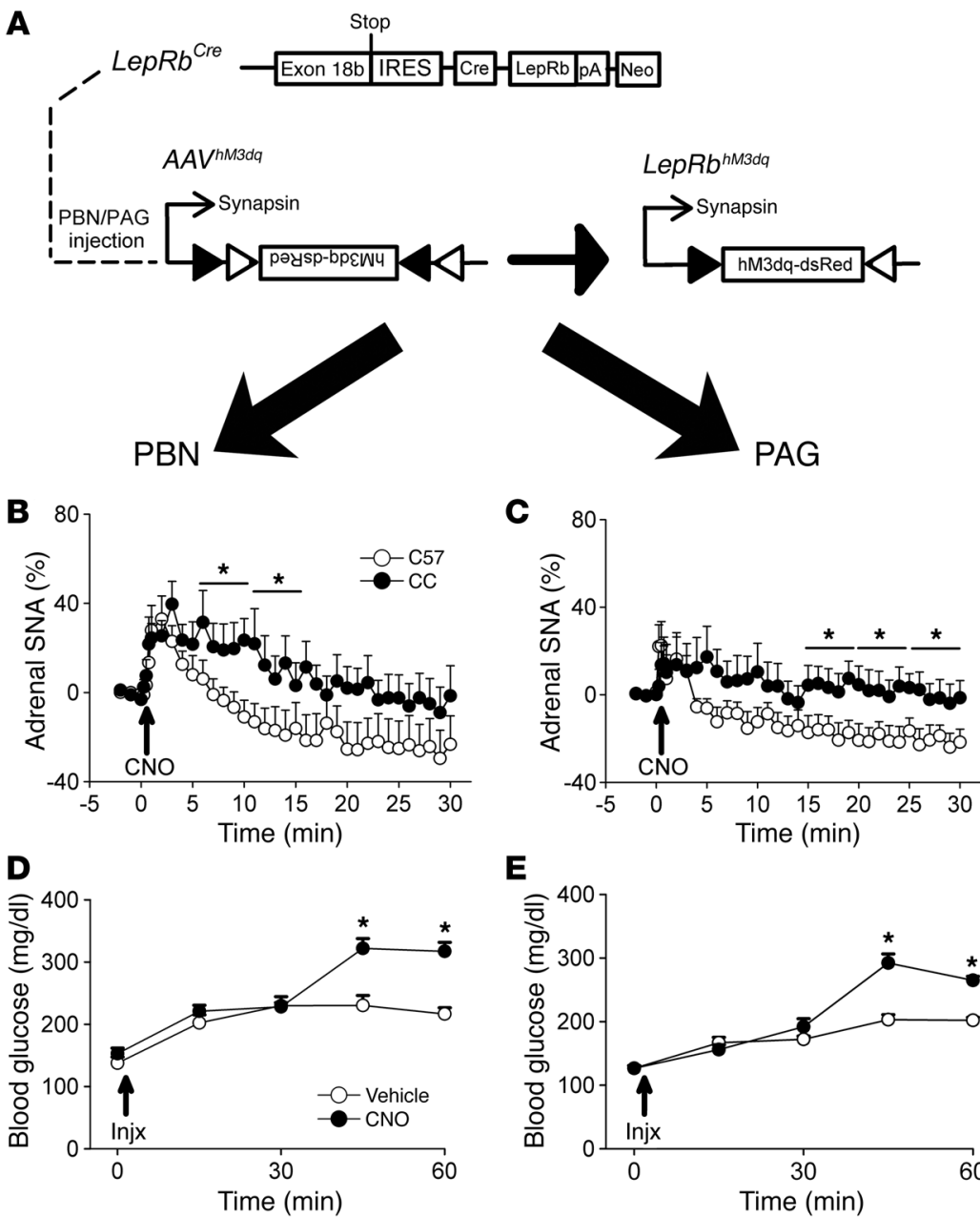

E

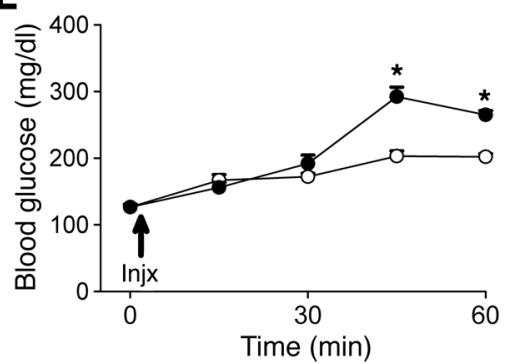

$\mathbf{F}$

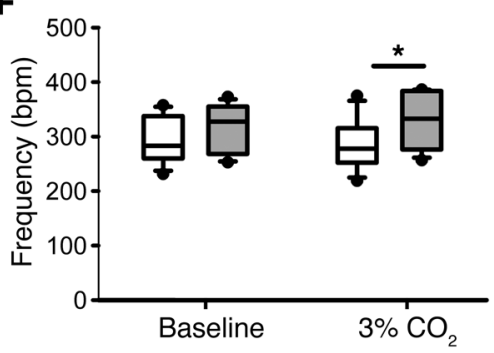

Figure 4. Activation of PAG ${ }^{\text {LepRb }}$ increases adrenal SNA, blood glucose, and respiratory responses to hypercapnia. (A) Lepr ${ }^{\text {Cre }}$ (CC) animals were injected with AAV-hM3Dq into the PBN (left panels) or PAC (right panels). (B and C) PBN (B) ( $n=8$, C57BL/6 [C57]; $n=6$, CC) and PAG (C) $(n=7$, C57BL/6; $n=9$, CC) AAV-hM3Dq-injected C57BL/6 or Lepr ${ }^{\text {cre }}$ (Cre) animals were treated with CNO during SNA recording. Data were broken down into 5-minute bins for analysis; data are plotted as mean $\pm \mathrm{SEM}$; ${ }^{*} P<0.05$. (D and E) PBN (D) or PAG (E) AAV-hM3Dq-injected Lepr ${ }^{\text {cre }}$ mice were injected with either CNO or vehicle and blood glucose sampled. Data are presented as mean \pm SEM; ${ }^{*} P<0.05$. (F) PAG-injected Lepr ${ }^{\text {re }}$ mice were treated with either CNO or vehicle, and respiratory frequency was monitored at baseline and during exposure to $3 \% \mathrm{CO}_{2}$ in a whole-body plethysmograph. Data are plotted as mean $\pm \mathrm{SEM}$; ${ }^{*} P<$ 0.05 . Data in panels $\mathbf{B}-\mathbf{E}$ were analyzed by 2 -way repeatedmeasures ANOVA with Fisher's LSD post hoc test. Data in panel $\mathbf{F}$ were analyzed using a 1-tailed $t$ test.

a constitutive AAV-TetTOX-GFP (which expresses tetanus toxin, preventing neurotransmitter release and effectively silencing all PBN neurons, plus GFP) in mice that also received injections of AAV-hM3Dq in the PBN of Lepr ${ }^{\mathrm{Cre}}$ mice (Figure 7A. As previously, $\mathrm{CNO}$ increased blood glucose concentrations in mice expressing hM3Dq in LepR $\mathrm{b}^{\mathrm{PAG}}$ neurons plus control virus in the PBN (Figure 7, B and C). In contrast, the hyperglycemic effect of $\mathrm{CNO}$ was diminished in mice expressing hM3Dq in LepRb ${ }^{\text {PAG }}$ neurons plus AAV-TetTOX-GFP in the PBN. Thus, PBN-mediated neurotransmission plays a crucial role in the control of blood glucose by LepRb ${ }^{\text {PAG }}$ neurons.

Thus, the withdrawal of leptin action from LepRb ${ }^{\text {PAG }}$ cells enhances glucose mobilization and other autonomic responses to noxious stimuli (pain, hypercapnia) that signal acutely increased metabolic demand, consistent with a crucial role for these cells in the brain system that promotes adequate glucose mobilization and SNS activity when metabolic requirements increase acutely during negative energy balance (Figure 7D). decreased leptin action on LepR $\mathrm{R}^{\mathrm{PAG}}$ cells enhances glucose mobilization during noxious stimuli (Figure 6G). Importantly, however, measures of pain perception and sensitivity were not altered in $\mathrm{LepRb}^{\mathrm{PAG}} \mathrm{KO}$ mice (Supplemental Figure 8), indicating that leptin action via the PAG controls glucose mobilization during noxious stimuli, but does not alter the threshold for noxious stimulus perception. Furthermore, the breathing frequency of LepRb ${ }^{\mathrm{PAC}} \mathrm{KO}$ mice was not different from that of controls in room air, but increased compared with control animals in the presence of $3 \%$ $\mathrm{CO}_{2}$ (Figure 6H), consistent with the notion that decreased leptin action on LepRb ${ }^{\mathrm{PAG}}$ cells enhances the autonomic hyperventilatory response to hypercapnia.

Our tracing experiments (Figures 2 and 3) suggest that projections from LepRb ${ }^{\text {PAG }}$ cells to the PBN could mediate the actions of these cells. To test this directly, we injected control AAV-GFP or

\section{Discussion}

We have identified a role for low leptin during negative energy balance in augmenting the autonomic response to noxious stimuli that signify acute unmet metabolic needs, thus ensuring that the drive to mobilize glucose is sufficient to overcome the additive effects of low SNS tone and depleted body energy stores (Figure 1A). Hence, this system can mitigate the detrimental effects of what would otherwise be diminished glucose mobilization during metabolic challenges in the face of the low SNS tone and depleted energy reserves that accompany negative energy balance.

Our findings also reveal the neural circuit that mediates this function (Figure 7D), beginning with LepRb ${ }^{\text {PAG }}$ neurons - the largest group of LepRb neurons in the brain stem, whose function was previously unknown $(6,8)$. Noxious stimuli (which indicate the need to mobilize glucose to support appropriate responses) 
A

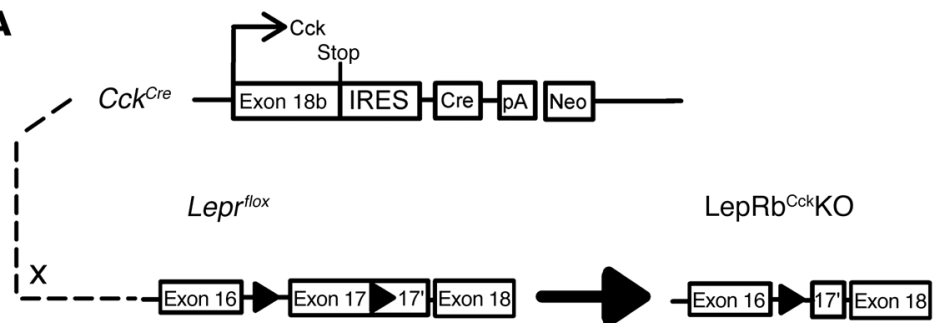

B

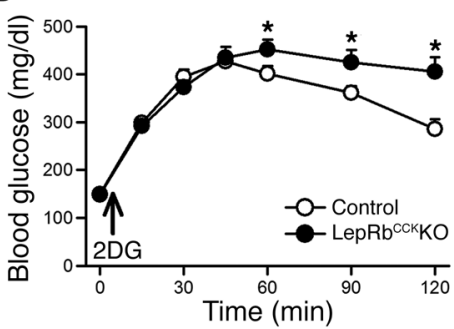

C

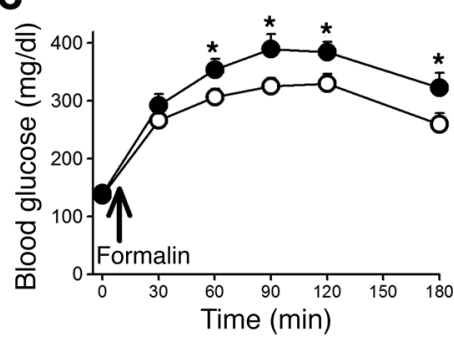

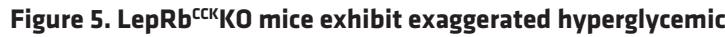
responses to formalin injection. (A) LepR ${ }^{\text {CCKK}} K O$ mice were generated by crossing $C c k^{c r e}$ onto the Lepr ${ }^{f l}$ background. (B) Lepr $r^{f l / f l}$ (control) $(n=12)$ and LepRb ${ }^{\text {ccKKO }}(n=10)$ mice were treated with $2 \mathrm{DC}(500 \mathrm{mg} / \mathrm{kg}$, i.p.) and monitored for blood glucose. (C) A separate group of animals was treated with intra-hind paw formalin (5\%, $20 \mu \mathrm{l})\left(n=17\right.$, control; $n=13$, LepRb $\left.{ }^{\text {ccKKO }}\right)$, and blood glucose was monitored. Data are plotted as mean $\pm \mathrm{SEM} ;{ }^{*} P<0.05$. Data in panels $\mathbf{B}$ and $\mathbf{C}$ were analyzed with 2-way repeated-measures ANOVA with Fisher's LSD post hoc test. activate LepRb ${ }^{\mathrm{PAg}}$ neurons, which project to the $\mathrm{PBN} \rightarrow \mathrm{VMN}$ circuit and promote SNS outflow and glucose mobilization. The withdrawal of leptin action from LepR ${ }^{\mathrm{PAG}}$ cells enhances glucose mobilization in response to noxious stimuli, revealing a role for these cells in ensuring adequate glucose mobilization in response to acute demands when leptin levels are low due to negative energy balance. In addition to the obvious survival benefit conferred by this system in the wild, it may also complicate the analysis of glycemic control, especially by inexperienced researchers who inadvertently provoke noxious stimuli while measuring blood glucose in fasted or leptin-deficient animals.

Interestingly, the time course with which the hM3Dq-mediated activation of LepRb ${ }^{\mathrm{PAG}}$ neurons augments blood glucose is delayed relative to some acute sympathetic responses to noxious stimuli (such as those that increase heart rate and blood pressure). This finding may indicate that the negative energy balance-mediated augmentation of glucose mobilization in response to noxious stimuli operates via a different, more slowly acting system and thus with a delayed time course compared with these other parameters. This would suggest that LepRb ${ }^{\mathrm{PAG}}$ neurons and their circuits play more important roles in response to stimuli that develop over or last for many minutes, thus indicating the need for more sustained glucose mobilization (rather than mediating the immediate response to noxious stimuli that might be adequately met with an initial burst of SNS activation). Alternatively, activation of LepR $\mathrm{B}^{\mathrm{PAG}}$ neurons or the circuits they subserve might require other contextual cues to mediate the more immediate augmentation of blood glucose.

Since activating LepRb ${ }^{\mathrm{PAG}}$ neurons or ablating LepRb in these cells also augments the ventilatory response to hypercapnia (which signals high metabolic demand relative to ventilation), LepRb ${ }^{\text {PAG }}$ cells presumably match ventilatory drive to the needs of acutely increased metabolic demand when leptin levels are low. These functions for LepR $\mathrm{B}^{\mathrm{PAG}}$ cells are consistent with the general role for the PAG in mediating sympathetic activation (23-25) and many autonomic responses to pain $(12,26)$ as well as the control of respiratory rate and the response to hypercapnia (19-22).

The PAG is a large nucleus, different regions of which play relatively distinct roles in autonomic and behavioral control. The ventral PAG receives projections primarily from the spinal cord and brain stem and mediates passive coping responses, such as freezing and decreasing blood pressure $(25,26)$. In contrast, the dorsal PAG (especially the caudal dorsal PAG) receives projections from sensory processing centers and mediates active coping responses, such as increased SNS output and flight (23-26). The majority of the PAG LepRb cells lie within this caudal dorsal region of the PAG, which also contains the vast majority of noxious stimulus-activated LepRb neurons. Due to the size of the PAG, it is not possible to target the entire length and depth of this nucleus by injection; we focused on the caudal dorsal regions with our injections, using 2 injections per side (4 injections total per mouse) to sufficiently target the relevant populations of LepRb ${ }^{\mathrm{PAG}}$ neurons. The role of LepRb $\mathrm{b}^{\mathrm{PAG}}$ cells in promoting SNS outflow, activity, glucose mobilization, and increased respiratory rate are consistent with the known output of this region. Since LepRb neurons in other areas of the PAG (and in adjoining structures, such as the DR) could mediate distinct processes, it will be interesting to define their functions as well.

This $\mathrm{LepRb}^{\mathrm{PAG}} \rightarrow \mathrm{PBN} \rightarrow \mathrm{VMN}$ circuit demonstrates multiple levels of specificity. Since it is engaged by the removal of leptin action, it is activated specifically during negative energy balance, when it is required to augment the drive to mobilize glucose. Also, it is activated only during times of acute need (i.e., it does not augment glucose mobilization in the absence of a noxious stimulus or another inciting event). Furthermore, the overall LepRb ${ }^{\text {PAG }} \rightarrow \mathrm{PBN} \rightarrow \mathrm{VMN}$ system is specific for stressors that indicate an acute metabolic challenge (e.g., noxious stimuli, elevated $\mathrm{CO}_{2}$, or in the case of the PBN, glucoprivation); this system is not activated during restraint stress (8) or in response to acute hypotension (data not shown), for instance.

While restraint stress does not activate LepRb ${ }^{\mathrm{PAG}}$ or LepRb ${ }^{\mathrm{PBN}}$ cells, it activates LepRb ${ }^{\mathrm{EW}}$ cells (27). Since LepRb is ablated from LepRb ${ }^{\mathrm{EW}}$ cells in LepRb ${ }^{\mathrm{CCK}} \mathrm{KO}$ mice, but the glycemic response to restraint stress is not altered in these animals (8), LepRb ${ }^{\mathrm{EW}}$ cells must modulate different outputs than do LepRb ${ }^{\text {PAG }}$ and LepRb ${ }^{\text {PBN }}$ cells. Indeed, projections from $\mathrm{EW}$ cells target the striatum $(28,29)$ rather than the VMN, suggesting a potential role for LepRb $\mathrm{RW}^{\mathrm{EW}}$ cells in modulating motivated behaviors, rather than metabolic fuel mobilization. 
A

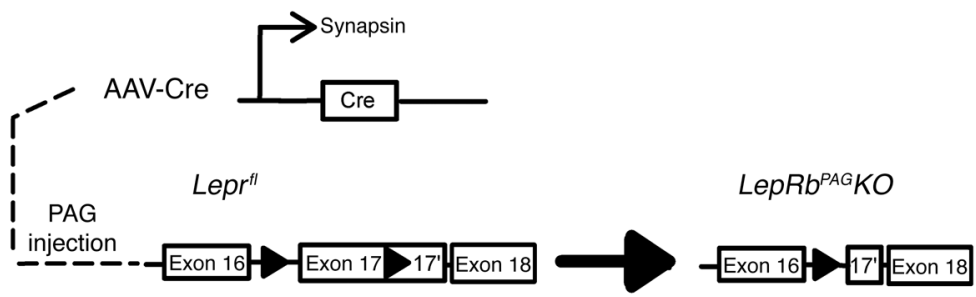

B

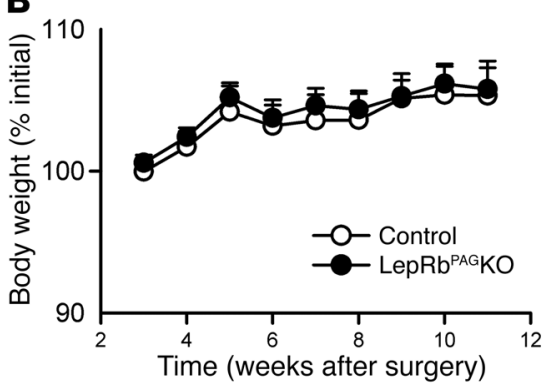

D
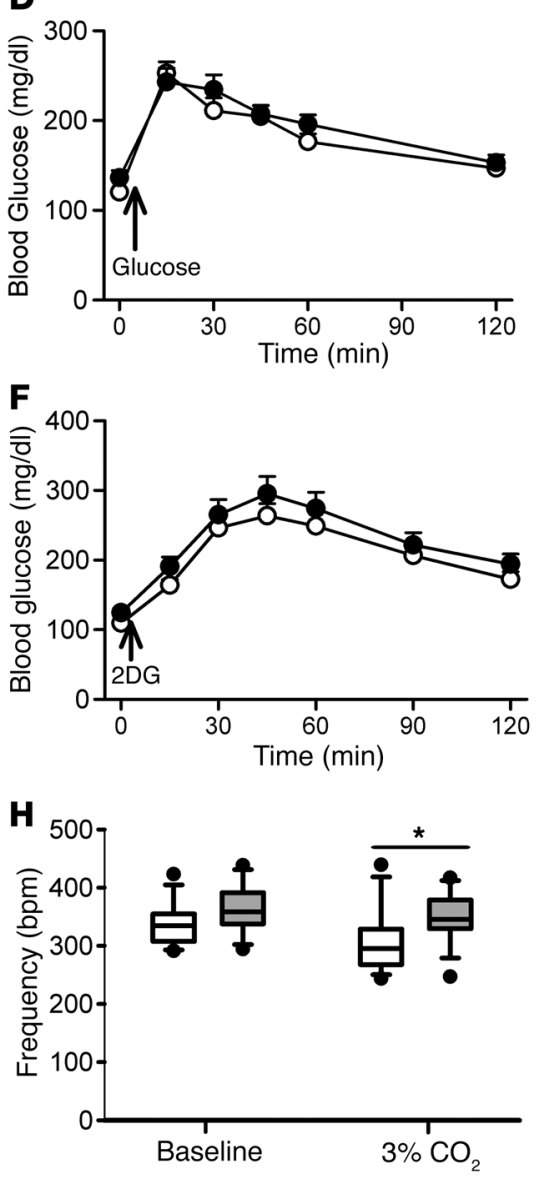

\section{C}
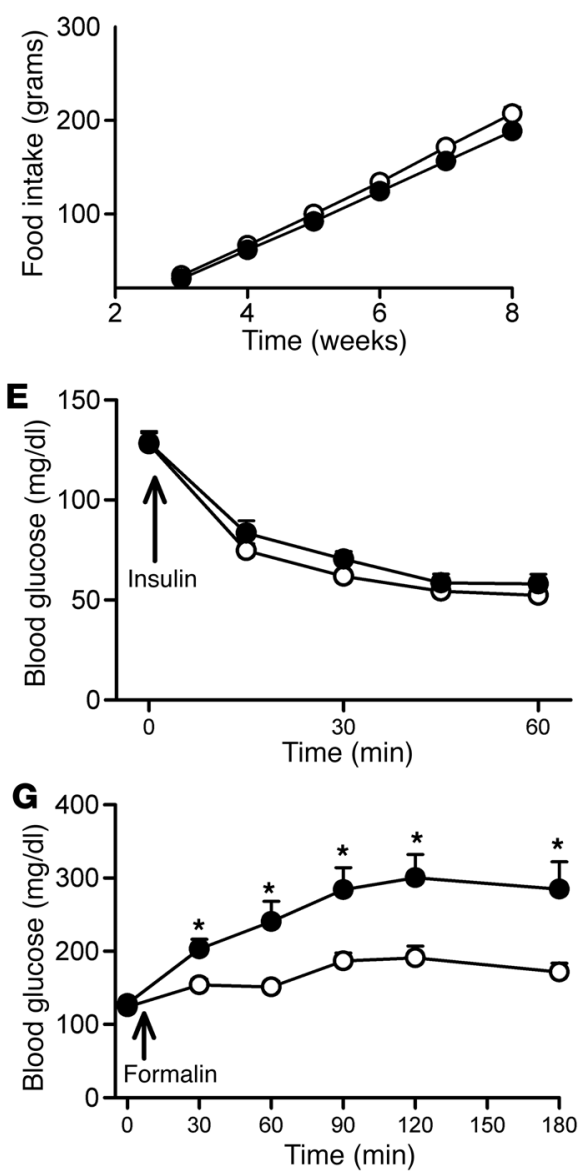

Figure 6. Deleting LepRb in the PAG exaggerates hyperglycemic responses to formalin and respiratory responses to hypercapnia. (A) Lepr $^{f / f l f l}$ mice on the Cre-inducible eGFP-L10a background were injected with AAV-Cre into the PAG to generate LepRb ${ }^{\text {PAC }} \mathrm{KO}$ mice $(n=13$,

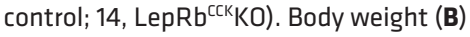
and food intake (C) of control-injected and LepRb ${ }^{\text {PAC }}$ KO mice were monitored weekly. In separate experiments, mice were treated with (D) glucose $(2 \mathrm{~g} / \mathrm{kg}$, i.p.), (E) insulin $(1.2 \mathrm{U} / \mathrm{kg}$, i.p.), (F) 2DG (250 mg/ $\mathrm{kg}$, i.p.), and (G) formalin (20 $\mu \mathrm{l}$, hind paw) and blood glucose concentrations were followed. (H) Respiratory rate was measured at baseline and following exposure to $3 \% \mathrm{CO}_{2}$. Data are plotted as mean $\pm \mathrm{SEM}$; ${ }^{*} P<0.05$. Data in panels B-G were analyzed using 2-way repeated-measures ANOVA with Fisher's LSD post hoc test. Data in panel $\mathbf{H}$ were analyzed using a 1-tailed $t$ test.

in the spinal cord do not directly synapse on LepRb $b^{\mathrm{PAg}}$ cells. In addition to noxious stimuli and hypercapnia, it is possible that other signals of acutely elevated metabolic demand that are integrated by the PAG might engage LepRb ${ }^{\mathrm{PAG}}$ neurons and their circuitry, as well.

Leptin modulates the $\mathrm{LepRb}^{\mathrm{PAG}} \rightarrow$ $\mathrm{PBN} \rightarrow \mathrm{VMN}$ circuit at 2 points - the PAG and the PBN. That the circuits downstream of LepRb ${ }^{\mathrm{PAG}}$ and LepR $\mathrm{b}^{\mathrm{PBN}}$ neurons overlap substantially makes teleological sense, since both circuits control similar physiologic end points (SNS outflow and glucose mobilization) and must therefore converge on the same output (the VMN in this case) to mediate their overlapping physiologic functions. This is not dissimilar to the convergence of DMH and arcuate nucleus (ARC) LepRb-expressing neurons on common pathways that control food intake and overall energy expenditure. While on the surface, it may seem redundant for leptin to act at multiple sites within a single circuit, leptin acts at least somewhat distinctly at these 2 sites; $\mathrm{PAG}^{\text {LepRb }}$ neurons do not contribute to the CRR to hyperglycemia and are only

The output of the LepRb $\mathrm{PAG}_{\mathrm{PABN}} \rightarrow \mathrm{VMN}$ circuit is largely specific for the augmentation of SNS outflow: this brain stem circuit does not represent a mechanism by which leptin modulates food intake, body weight, or metabolism at baseline, since none of these parameters were altered in LepRb ${ }^{\mathrm{CCK}} \mathrm{KO}$ or LepRb ${ }^{\mathrm{PAG}} \mathrm{KO}$ mice. Furthermore, our data reveal that leptin action on LepRb ${ }^{\mathrm{PAG}}$ cells does not alter noxious stimulus perception or responding, since the tailflick and withdrawal responses are not changed in the LepR $\mathrm{Rb}^{\mathrm{PAG}} \mathrm{KO}$ mice. This is consistent with our finding that pain-sensing neurons involved in responding to noxious stimuli. Thus, leptin action on LepRb $b^{\mathrm{PAG}}$ cells does not alter the CRR to hypoglycemia, whereas PBN leptin action does.

The expression of LepRb in the PAG (in addition to the PBN) suggests that PBN LepRb on its own may not adequately augment SNS activation and glucose mobilization in response to noxious stimuli. If so, this could reflect the need for cell-autonomous leptin action to modulate the cellular response to the noxious input. While silencing all PBN neurons blocked the hyperglyce- 
A
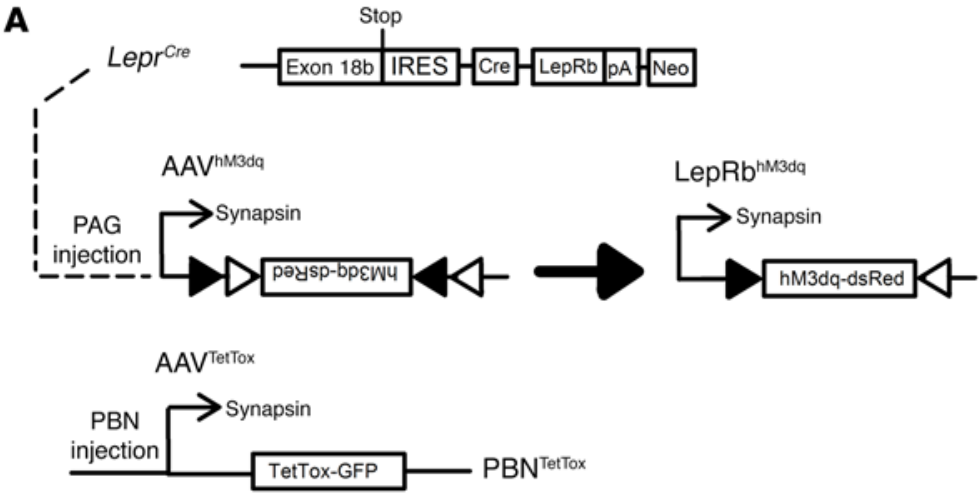

B

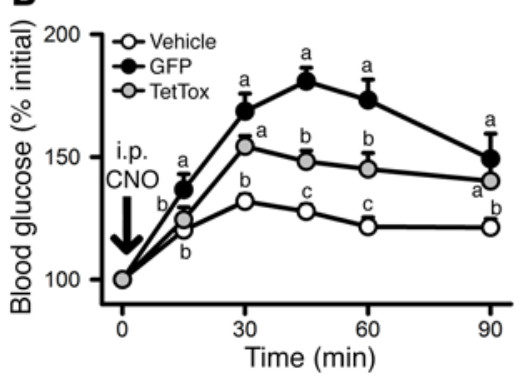

C

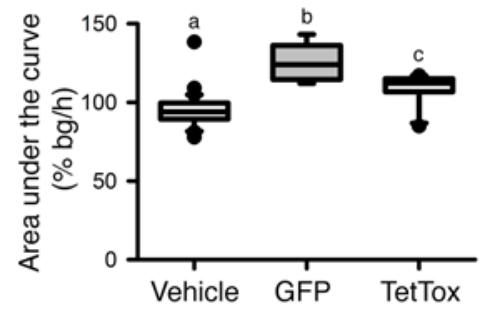

Figure 7. PAG ${ }^{\text {LepRb }}$ neurons act via the PBN to increase blood glucose. (A) Lepr ${ }^{\text {cre }}$ mice were injected with AAVhM3dq in the PAC and either a GFP-expressing control virus $(n=7)$ or AAV-TetTOX-GFP $(n=10)$ to constitutively express GFP or GFP plus TetTOX in the PBN, respectively. All mice were randomly assigned to receive vehicle control or CNO; mice received the other treatment the following week (B and $\mathbf{C}$ ). Glycemic responses following vehicle injection were not different between groups and were thus combined into a single control group. (D) Our data demonstrate that PAG ${ }^{\text {LepRb }}$ neurons act via the PBN to mobilize glucose and increase adrenal SNA when activated by noxious stimuli such as pain and hypercapnia. In the presence of sufficient energy stores, leptin inhibits these neurons to limit sympathetic activation and hyperglycemia to appropriate levels during the response to noxious stimuli. When energy stores are depleted, however, loss of leptin inhibition of PAG LepRb neurons augments sympathetic and glycemic responses to noxious stimuli to ensure a sufficient glucose mobilization in response to noxious stimuli, despite reduced overall sympathetic tone and the depletion of energy stores. Data are plotted as mean $\pm S E M$; groups with different letters are significantly different. $P<0.05$. Data in panel $\mathbf{B}$ were analyzed using 2-way repeated-measures ANOVA with Fisher's LSD post hoc test. Data in panel $\mathbf{C}$ were analyzed using 1-way ANOVA with Fisher's LSD post hoc test.

D

$\underline{\text { Normal energy stores }}$

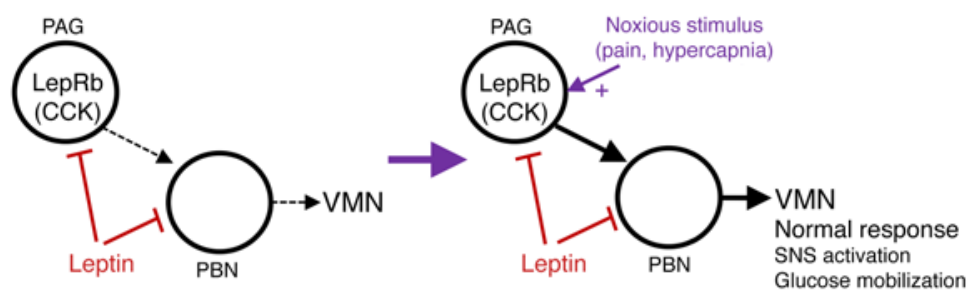

Depleted energy stores

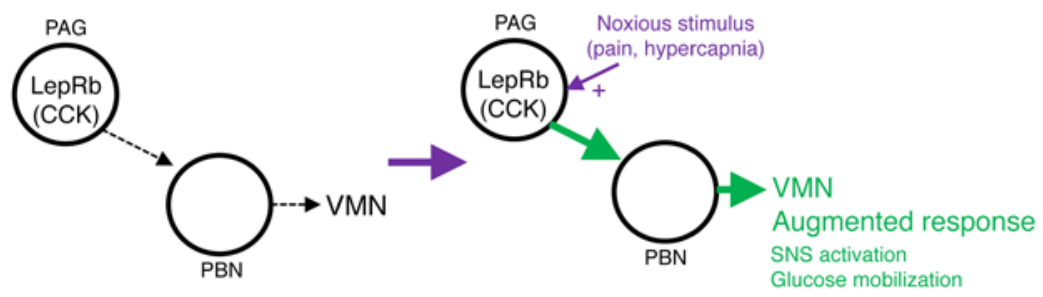

neither does leptin alter that activity of LepRb $b^{\text {PAG }}$ neurons in ex vivo slice preparations (data not shown). We previously observed that leptin inhibits LepRb ${ }^{\text {PBN }}$ cells under low-glucose conditions (8), but we were unable to detect leptin-mediated inhibition of these cells in normal glucose, suggesting that a relevant activating stimulus might be required to observe the inhibitory effect of leptin on these cells. Hence, the effect of leptin on LepRb ${ }^{\text {PAG }}$ cells might also be detectable only during activation of the neurons by other stimuli (e.g., by pain or hypercapnia). Unfortunately, it is not possible to stimulate ex vivo slice preparations with pain or hypercapnia, since the neurons that initially detect these stimuli lie remote to the LepRb ${ }^{\text {PAG }}$ cells. Thus, leptin might inhibit LepRb ${ }^{\mathrm{PAg}}$ neurons, but technical issues prevent us from detecting this change in ex vivo slices. Since LepRb ${ }^{\text {PAG }}$ neurons are largely glutamatergic (data not shown), the activation of

mic effect of activating LepRb ${ }^{\text {PAG }}$ cells, silencing LepRb ${ }^{\text {PBN }}$ cells did not (data not shown); thus LepRb ${ }^{\text {PAG }}$ neurons must activate non-LepRb PBN $\rightarrow$ VMN cells as well as LepRb ${ }^{\text {PBN }}$ cells to enhance glucose mobilization in response to noxious stimuli. Indeed, formalin activates a large number of non-LepRb PBN neurons that lie in the vicinity of LepRb ${ }^{\mathrm{PBN}}$ neurons, consistent with the notion that LepRb ${ }^{\mathrm{PBN}}$ cells represent only a subset of the PBN cells that make up the circuit downstream of LepRb ${ }^{\text {PAG }}$ neurons.

While the similar results of activating LepRb ${ }^{\text {PAG }}$ neurons and removing LepRb from these cells suggests that the lack of leptin signaling activates LepRb ${ }^{\mathrm{PAG}}$ neurons (i.e., that leptin inhibits LepRb ${ }^{\text {PAG }}$ neurons), leptin and fasting each fail to detectably alter the activity of LepRb ${ }^{\text {PAG }}$ cells by c-Fos immunohistochemistry; these cells by leptin could also stimulate an inhibitory interneuron to mediate the observed effects. It is also possible that leptin does not directly or acutely alter the firing rate of LepRb ${ }^{\text {PAG }}$ neurons, but rather mediates a transcriptional change that alters the magnitude of the response evoked by activating stimuli. Additionally, it is theoretically possible that the PAG could contain multiple subtypes of LepRb neurons.

Despite the power and utility of the genetic tools that we have employed to dissect the function of LepRb ${ }^{\text {PAG }}$ neurons, it must be recognized that these manipulations are artificial and extreme; the level of activation, silencing, or degree of leptin insensitivity that these tools produce is unlikely to be recapitulated under physiologic circumstances. Nonetheless, the consistency of the 
results produced by the various approaches undertaken suggests the veracity of our conclusions.

In addition to revealing the function of the largest population of LepRb neurons in the brain stem (LepRb ${ }^{\mathrm{PAG}}$ neurons), which had not, to our knowledge, previously been studied, our findings define a new component of the response to fasting and a new physiologic role for leptin: in addition to the hypothalamically mediated energy-sparing neuroendocrine response to starvation, low leptin augments the activation of a LepRb $b^{\mathrm{PAG}} \rightarrow \mathrm{PBN} \rightarrow \mathrm{VMN}$ circuit that ensures adequate SNS drive and glucose mobilization to meet the metabolic needs associated with noxious stimuli or hypoglycemia during negative energy balance. In the future, it will be important to identify other stimuli that act via this system to augment SNS outflow during negative energy balance as well as to define the detailed mechanisms by which this circuit mediates glucose mobilization.

\section{Methods}

Animals. All mice were provided with food and water ad libitum unless noted otherwise and kept in a temperature-controlled room on a 12-hour light/12-hour dark cycle. All experiments were carried out using approximately equal numbers of male and female mice. Animals were processed and studied in the order of their randomly assigned ear tags. Investigators were blinded to the genotype and/or treatment for all studies. All mice were genotyped via PCR across the potentially mutated region prior to study. C57BL/6 animals originated from Jackson Laboratories, but all other mice were bred within our colony. Lepr ${ }^{\mathrm{Cre} / \mathrm{Cre}}$ mice were on a C57BL/6 background, as previously described $(8,30-32)$. LepRb ${ }^{\text {eGFP-L10a }}$ mice were generated by interbreeding compound homozygous Lepr ${ }^{\text {Cre/Cre }}$ Rosa26 $6^{\text {eGFP-L10a/eGFP-L10a }}$ mice. LepRb ${ }^{\text {eYFP }}$ mice were generated by interbreeding compound homozygous

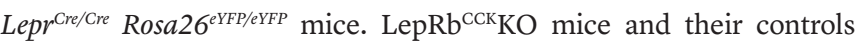
were generated by breeding male $C c k^{C r e} L e p r^{f / / f l}$ mice to female $L e p r^{f / f l}$ mice. Lepr $r^{f / f l}$ mice for AAV-Cre injection were generated by interbreeding homozygous Leprlflfl mice. When possible, animals were randomly assigned to groups and investigators were blind to treatment.

Reagents. The AAV-map2c-dsRed was developed by cloning the coding sequences for the map2c-dsRed fusion protein (in reverse orientation) into the base Cre-inducible AAV vector, using SacI and NheI restriction sites. AAV-map2c-dsRed stocks were prepared at the University of North Carolina (UNC) Vector Core (Chapel Hill, North Carolina, USA). The AAV-Syn-GFP and AAV-TVA+G helper viruses were previously described $(33,34)$; viral stocks were prepared at the UNC Vector Core. The Rabies- $\Delta \mathrm{G}-\mathrm{mCherry}$ was as previously described $(18,34)$ and was generated at the University of Michigan Viral Vector Core with the support of the Molecular Genetics core of the Michigan Diabetes Research Center (University of Michigan). AAV-hM3dq, AAV-mCherry, AAV-GFP, and AAV-Cre were purchased from the UNC vector core. AAV-TetTox-GFP was from the Stanford University Viral Vector Core (Stanford, California, USA).

Perfusion and immunohistochemistry. Brains were collected and processed as previously described (8). To reveal leptin-responsive neurons by pSTAT3, leptin was administered ( $5 \mathrm{mg} / \mathrm{kg}$, i.p.) 2 hours prior to perfusion. For c-Fos studies, mice were exposed to a given stimulus 90 minutes prior to perfusion. After either stimulus exposure or leptin administration, mice were anesthetized with avertin prior to transcardial perfusion with PBS followed by $10 \%$ formalin. Brains were removed and placed into $10 \%$ formalin overnight, followed by
$30 \%$ sucrose for at least 1 day. Brains were cut into $30-\mu \mathrm{m}$ sections on a freezing microtome in 4 series and stored in antifreeze solution (25\% ethylene glycol, $25 \%$ glycerol). Sections were washed with PBS and then treated sequentially with $1 \%$ hydrogen peroxide $/ 0.5 \%$ sodium hydroxide, $0.3 \%$ glycine, and $0.03 \%$ sodium dodecyl sulfate. Pretreatment was followed by an hour in blocking solution (PBS containing 0.1\% Triton X-100, 3\% normal donkey serum), then overnight incubation in blocking solution containing rabbit anti-c-Fos (sc-52, Santa Cruz Biotechnology Inc., 1:1000) and/or chicken anti-GFP (GFP-1020, Aves, 1:1000), rabbit anti-dsRed (632496, Living Colors, 1:1000), rabbit anti-pSTAT3 (91455, Cell Signaling Technology, 1:500), or rabbit anti-calcitonin gene-related peptide (anti-CGRP) (PC205L, Millipore, 1:1000) at room temperature. The next day, the sections were incubated with fluorescent secondary antibodies (Molecular Probes, 1:200) or with biotinylated secondary antibody (Jackson Immunoresearch, 1:200) followed by ABC amplification (Vector Laboratories, 1:500) and diaminobenzadine (Thermo Scientific) reaction. The sections treated with biotinylated secondary antibody were exposed to avidin/biotin complex solution followed by diaminobenzadine reaction. The sections were mounted onto slides coverslipped with Fluoromount-G (Southern Biotech). Images were collected on an Olympus Bx53 microscope, and the number of immunoreactive cells were counted using Adobe photoshop. The number of c-Fos-positive nuclei colocalizing with GFP-expressing cells were counted, along with total GFP-containing cells, and were expressed as a percentage of all GFP-expressing cells within the region of interest.

Phenotypic studies. Body weight and food intake were monitored weekly in animals starting at 3 weeks after surgery. Glycemic measures, including glucose tolerance test (GTT) $(2 \mathrm{~g} / \mathrm{kg}$ body weight, i.p.), insulin tolerance test (ITT) (1.2 unit/kg body weight, Humulin, Eli Lilly, i.p.), 2DG (Sigma-Aldrich) challenge ( 250 or $500 \mathrm{mg} / \mathrm{kg}$ body weight, ip), and formalin challenge $(20 \mu \mathrm{l} ; 5 \%$ formalin, intra-hind paw) were determined in 12- to 18-week-old animals by analyzing tail vein blood with a OneTouch Ultra 2 glucometer (Johnson \& Johnson). For formalin challenge, mice were not included in the study unless there was visual inspection confirming inflammation of the hind paw to control for a missed injection. To measure leptin-induced pSTAT3, animals were fasted for 24 hours prior to leptin administration. For fasting modulation of hyperglycemic responses to intra-hind paw formalin injection, mice were fasted for 12 hours. For DREADD studies, CNO (Tocris Biosciences) was administered via i.p. injection at 0.3 $\mathrm{mg} / \mathrm{kg}$ body weight.

Stereotaxic injections. Eight- to fourteen-week-old animals were anesthetized with $1.5 \%-2 \%$ isoflurane in preparation for craniotomy. The skull was exposed, bregma and lambda were leveled, a hole was drilled, and we injected the contents of a pulled pipette at the coordinates of our target for approximately $25 \mathrm{nl} / \mathrm{min}$. For the PBN, $100 \mathrm{nl}$ of virus was injected at anteroposterior (AP) -4.85 , mediolateral (ML) \pm 1.2, and dorsoventral (DV) -3.3. For the PAG, $100 \mathrm{nl}$ of virus was injected at 4 separate coordinates per animal (AP: -4.9, ML: 0, DV: 0.7; AP: -4.8, ML: \pm 0.6, DV: 0.95; AP: -4.7, ML: 0, DV: 0.8). For the VMN, 50 $\mathrm{nl}$ was injected at AP: -1.1 , ML: \pm 0.35 , DV: -3.3 . After 5 minutes, to allow the virus to diffuse into the brain, the pipette was raised slowly out of the hole in the skull, the hole in the skull was filled with bone wax, and the skin was closed with surgical sutures (Henry Schein). Analgesics were administered prophylactically to all mice to prevent postsurgical pain. The animals were allowed 4 weeks to recover from 
surgery before experimental manipulation. At 3 weeks after surgery, the animals were individually housed for experiments that included body weight, food intake, and glycemic analyses. For tracing studies, mice were euthanized at different times after injection depending on the virus (rabies, 1 week; map2c-dsRed/Syn-GFP, 2 weeks). We used fluorescent reporters to confirm injection of the virus into the target brain region in all studies; if the fluorescence was not observed within the region of interest or leaked into another region containing LepRb neurons, these cases were omitted from analyses. In the case of rabies tracing, the VMN injection site was confirmed by visualizing inflammation and the needle track 1 week after injection, since the pseudotyped rabies virus requires TVA expression for initial viral entry and TVA expression was restricted to the PBN.

Adrenal SNA. Adrenal SNA was assessed using multifiber nerve recording. Mice (14 to 20 weeks old) that received the DREADDhM3dq virus in either the PAG or PBN were initially anesthetized with i.p. administration of ketamine $(91 \mathrm{mg} / \mathrm{kg}$ body weight) and xylazine $(9.1 \mathrm{mg} / \mathrm{kg}$ body weight). Intubation with PE-50 was performed to provide an unimpeded airway for the mouse to spontaneously breathe $\mathrm{O}_{2}$-enriched room air. Micro-renathane tubing (MRE-40, Braintree Scientific) was inserted into the right jugular vein for infusion of the sustaining anesthetic agent: $\alpha$-chloralose (initial dose: 12 $\mathrm{mg} / \mathrm{kg}$, then sustaining dose of $6 \mathrm{mg} / \mathrm{kg} / \mathrm{h}$ ). Another MRE-40 tubing was inserted into the left carotid artery for continuous measurement of arterial pressure and heart rate. Core body temperature was maintained at $37.5^{\circ} \mathrm{C}$. A retroperitoneal incision was made to gain access to the preganglionic nerve fibers that innervate the left adrenal gland. A single multifiber nerve filament was carefully placed on a bipolar platinum-iridium wire (40 gauge, A-M Systems) and then sealed with silicone gel (Kwik-Sil, WPI). The adrenal nerve signal (AdSNA) was amplified $10^{5}$ times, with low- and high-frequency filter cutoffs at 100 and $1 \mathrm{Khz}$, respectively. The amplified and filtered signal was routed to a data acquisition system (MacLab Model 8s), which counted the number of nerve spikes that exceeded a threshold level set just above the noise level each second. After allowing all hemodynamic parameters to stabilize, a slow i.v. infusion of $\mathrm{CNO}$ was given over 60 seconds and adrenal SNA was continuously monitored for the next 30 minutes. Next, a slow infusion of vehicle (hydroxypropyl $\beta$ cyclodextrin; $5 \mu \mathrm{l} / \mathrm{g}$ body weight, i.v.) was given over 60 seconds and the adrenal SNA was continuously measured for next 30 minutes. Background noise was excluded in the assessment of adrenal SNA by correcting for postmortem activity.

Behavioral analyses. Activity of 18- to 22-week-old mice was determined in the open-field test. The mice were introduced to the openfield chamber for 2 hours prior to experimentation. On the following day, the mice were introduced to the chamber for 1 hour of baseline measurement followed by activity recording 1 hour after injection. Data were analyzed as the total number of counts over this 1-hour period. Tail flick and hind paw latency were tested as previously described using a tail-flick test analgesia meter (IITC Life Science) (35). For the tail-flick test, mice were individually restrained during the test to determine the latency to remove the tail away from a 75-degree light on 3 separate instances; the average of the 3 instances was used as an individual data point. For the hind paw test, the mice were allowed to acclimate to a Plexiglass chamber for 1 hour prior to testing. The 75-degree light was then concentrated on the hind paw of an immobile mouse, and the latency for the hind paw to move away from the light was recorded (36). As with the tail-flick test, 3 separate instances of this test were recorded and averaged for an individual data point.

Respiratory measurement. Baseline ventilation and the ventilatory response to hypercapnia were measured in freely moving, conscious 14- to 18-week-old mice using a whole-body plethysmograph (Emka Technologies). Gas tanks of $\mathrm{O}_{2}, \mathrm{~N}_{2}$, and $\mathrm{CO}_{2}$ were connected to the plethysmograph and digitally controlled by the plethysmograph to deliver gas mixtures into each plethysmograph mouse chamber. Each mouse was placed inside the chamber for 20 minutes to acclimate. After acclimation, baseline ventilation was measured while the mice were exposed to room air (e.g., $21 \% \mathrm{O}_{2}, 79 \% \mathrm{~N}_{2}, 0 \% \mathrm{CO}_{2}$ ). To determine the ventilatory response to hypercapnia, the $\mathrm{CO}_{2}$ concentration inside the chamber was elevated to $3 \%$ for periods of 4 minutes, separated by 5 -minute recovery periods in room air (similar to ref. 37 ), while real-time breathing frequency was recorded. As $\mathrm{CO}_{2}$ was increased, $\mathrm{N}_{2}$ was decreased to allow $\mathrm{O}_{2}$ levels to be stabilized at $21 \%$. In total, mice remained in the plethysmograph for approximately an hour. All respiratory measures were recorded between 8 am and $1 \mathrm{pm}$. During recording, mice were continuously monitored by the investigator to ensure that they stayed awake (as the ventilation response is blunted by sleep; ref. 37). If a mouse fell asleep, the investigator aroused the animal by gently tapping on the chamber.

Statistics. Statistical methods were not used to predetermine sample sizes, but our sample sizes are similar to those in previously published studies using similar approaches (8, 17, 37-39). Sample sizes for groups that involved stereotaxic injection of specific populations of cells were $40 \%$ larger than for other groups to account for a 50\%-60\% bilateral hit rate. c-Fos data and activity data were analyzed by 1-tailed $t$ test. Blood glucose data were analyzed by 2-way repeated-measures ANOVA with Fisher's least significant difference (LSD) post hoc test. Adrenal SNA data were analyzed by 1-tailed $t$ test of 5-minute bins across the experiment. No data were removed unless the injection was a miss or the animal was sick or injured at the time of the experiment (loss of $>10 \%$ body weight). Data that did not fall under a normal distribution or equal variance were log transformed, then reanalyzed. Significance was set at $P \leq 0.05$. All data were analyzed using SigmaStat (Systat).

Study approval. The procedures included in this manuscript were approved by the appropriate Committees on the Use and Care of Animals at the University of Michigan and the University of Iowa.

\section{Author contributions}

All authors edited the manuscript. JNF designed and contributed to the performance of all experiments, interpreted the data, ran the statistical tests, and wrote the manuscript. MGM also designed all experiments, interpreted the data, and wrote the manuscript. DA and RJS helped design and ran the respiratory measurement testing shown in Figure 4 and Figure 6. WP designed and constructed the AAV-map2c-dsRed used for Figure 2. CP helped design and run the glucose tests shown in Figure 4 and Figure 5. TL contributed to the design and execution of the rabies tracing experiments. PBG aided in studies of PAG LepRb neuron regulation. JS helped run LepR $\mathrm{B}^{\mathrm{PAG}} \mathrm{KO}$ experiments and validation of our mouse models used in Figure 6 and Supplemental Figure 7. MJ helped run PAG and PBN DREADD experiments used for Figure 4. DAM and KR helped design and run adrenal SNA experiments shown in Figure 4. MBA designed and constructed the rabies helper virus 
used in Figure 2 and Figure 3. JH and EF helped design and run pain behavior tests shown in Supplemental Figure 8. DPO helped design and run experiments and helped with data interpretation.

\section{Acknowledgments}

We thank MedImmune Inc. for the generous gift of leptin, Karl Deisseroth for the use of AAV-SynGFP, members of the Myers and Olson labs for helpful discussions, and the University of Michigan Vector Core. Research support was provided by the Michigan Diabetes Research Center (NIH P30 DK020572, including the Molecular Genetics, Animal Phenotyping, and Clinical Cores), the American Diabetes Association (to MGM and JNF), the Marilyn H. Vincent Foundation (to MGM), the American Heart Association (to KR), the University of Iowa Center for Hypertension Research and the University of Iowa Fraternal Order of Eagles Diabetes Research Center (to KR), and the NIH (DK098853 to MGM; DK098833 to JNF; HL084207 to KR).

Address correspondence to: Martin G. Myers Jr., Departments of Internal Medicine and Physiology, University of Michigan, 1000 Wall Street, 6317 Brehm Tower, Ann Arbor, Michigan 48105, USA. Phone: 734.647.9515; Email: mgmyers@umich.edu.
1. Friedman JM, Halaas JL. Leptin and the regulation of body weight in mammals. Nature. 1998;395(6704):763-770.

2. Ahima RS, et al. Role of leptin in the neuroendocrine response to fasting. Nature. 1996;382(6588):250-252.

3. Schwartz MW, et al. Cooperation between brain and islet in glucose homeostasis and diabetes. Nature. 2013;503(7474):59-66.

4. Flak JN, Myers MG. Minireview: CNS Mechanisms of Leptin Action. Mol Endocrinol. 2016;30(1):3-12.

5. Ring LE, Zeltser LM. Disruption of hypothalamic leptin signaling in mice leads to early-onset obesity, but physiological adaptations in mature animals stabilize adiposity levels. J Clin Invest. 2010;120(8):2931-2941.

6. Patterson CM, Leshan RL, Jones JC, Myers MG. Molecular mapping of mouse brain regions innervated by leptin receptor-expressing cells. Brain Res. 2011;1378:18-28.

7. Scott MM, et al. Leptin targets in the mouse brain. JComp Neurol. 2009;514(5):518-532.

8. Flak JN, et al. Leptin-inhibited PBN neurons enhance responses to hypoglycemia in negative energy balance. Nat Neurosci. 2014;17(12):1744-1750.

9. Garfield AS, et al. A parabrachial-hypothalamic cholecystokinin neurocircuit controls counterregulatory responses to hypoglycemia. Cell Metab. 2014;20(6):1030-1037.

10. Behbehani MM. Functional characteristics of the midbrain periaqueductal gray. Prog Neurobiol. 1995;46(6):575-605.

11. Heinricher MM, Tavares I, Leith JL, Lumb BM. Descending control of nociception: Specificity, recruitment and plasticity. Brain Res Rev. 2009;60(1):214-225.

12. Mason P. Deconstructing endogenous pain modulations. J Neurophysiol. 2005;94(3):1659-1663.

13. Lovick TA. Pro-nociceptive action of cholecystokinin in the periaqueductal grey: a role in neuropathic and anxiety-induced hyperalgesic states. Neurosci Biobehav Rev. 2008;32(4):852-862.

14. Porro CA, Cavazzuti M. Spatial and temporal aspects of spinal cord and brainstem activation in the formalin pain model. Prog Neurobiol. 1993;41(5):565-607.

15. Tjølsen A, Berge OG, Hunskaar S, Rosland JH, Hole K. The formalin test: an evaluation of the method. Pain. 1992;51(1):5-17.

16. Hermanson O, Larhammar D, Blomqvist A. Preprocholecystokinin mRNA-expressing neu- rons in the rat parabrachial nucleus: subnuclear localization, efferent projection, and expression of nociceptive-related intracellular signaling substances. J Comp Neurol. 1998;400(2):255-270.

17. Allison MB, Patterson CM, Krashes MJ, Lowell BB, Myers MG, Olson DP. TRAP-seq defines markers for novel populations of hypothalamic and brainstem LepRb neurons. Mol Metab. 2015;4(4):299-309.

18. Wall NR, Wickersham IR, Cetin A, De La Parra M, Callaway EM. Monosynaptic circuit tracing in vivo through Cre-dependent targeting and complementation of modified rabies virus. Proc Natl Acad Sci U S A. 2010;107(50):21848-21853.

19. Lopes LT, Patrone LG, Bícego KC, Coimbra NC, Gargaglioni LH. Periaqueductal gray matter modulates the hypercapnic ventilatory response. Pflugers Arch. 2012;464(2):155-166.

20. Brannan S, et al. Neuroimaging of cerebral activations and deactivations associated with hypercapnia and hunger for air. Proc Natl Acad Sci US A. 2001;98(4):2029-2034.

21. Kramer JM, Nolan PC, Waldrop TG. In vitro responses of neurons in the periaqueductal gray to hypoxia and hypercapnia. Brain Res. 1999;835(2):197-203.

22. Teppema LJ, Veening JG, Kranenburg A, Dahan A, Berkenbosch A, Olievier C. Expression of c-fos in the rat brainstem after exposure to hypoxia and to normoxic and hyperoxic hypercapnia. J Comp Neurol. 1997;388(2):169-190.

23. Carrive P. The periaqueductal gray and defensive behavior: functional representation and neuronal organization. Behav Brain Res. 1993;58(1-2):27-47.

24. Walker DL, Cassella JV, Lee Y, De Lima TC, Davis $\mathrm{M}$. Opposing roles of the amygdala and dorsolateral periaqueductal gray in fear-potentiated startle. Neurosci Biobehav Rev. 1997;21(6):743-753.

25. Bandler R, Keay KA, Floyd N, Price J. Central circuits mediating patterned autonomic activity during active vs. passive emotional coping. Brain Res Bull. 2000;53(1):95-104.

26. Linnman C, Moulton EA, Barmettler G, Becerra L, Borsook D. Neuroimaging of the periaqueductal gray: state of the field. Neuroimage. 2012;60(1):505-522.

27. Xu L, et al. Integration of stress and leptin signaling by CART producing neurons in the rodent midbrain centrally projecting Edinger-Westphal nucleus. Front Neuroanat. 2014;8:8.

28. Bachtell RK, et al. The Edinger-Westphallateral septum urocortin pathway and its rela- tionship to alcohol consumption. JNeurosci. 2003;23(6):2477-2487.

29. Bittencourt JC, Vaughan J, Arias C, Rissman RA, Vale WW, Sawchenko PE. Urocortin expression in rat brain: evidence against a pervasive relationship of urocortin-containing projections with targets bearing type $2 \mathrm{CRF}$ receptors. J Comp Neurol.1999;415(3):285-312.

30. Leshan RL, et al. Ventral tegmental area leptin receptor neurons specifically project to and regulate cocaine- and amphetamine-regulated transcript neurons of the extended central amygdala. J Neurosci. 2010;30(16):5713-5723.

31. Leinninger GM, et al. Leptin acts via leptin receptor-expressing lateral hypothalamic neurons to modulate the mesolimbic dopamine system and suppress feeding. Cell Metab. 2009;10(2):89-98

32. Louis GW, Leinninger GM, Rhodes CJ, Myers MG. Direct innervation and modulation of orexin neurons by lateral hypothalamic LepRb neurons. J Neurosci. 2010;30(34):11278-11287.

33. Carter ME, Soden ME, Zweifel LS, Palmiter RD. Genetic identification of a neural circuit that suppresses appetite. Nature. 2013;503(7474):111-114.

34. Meek TH, et al. Functional identification of a neurocircuit regulating blood glucose. Proc Natl Acad Sci U S A. 2016;113(14):E2073-E2082.

35. Hinder LM, Vincent AM, Hayes JM, McLean LL, Feldman EL. Apolipoprotein E knockout as the basis for mouse models of dyslipidemia-induced neuropathy. Exp Neurol. 2013;239:102-110.

36. O'Brien PD, Hur J, Hayes JM, Backus C, Sakowski SA, Feldman EL. BTBR ob/ob mice as a novel diabetic neuropathy model: Neurological characterization and gene expression analyses. Neurobiol Dis. 2015;73:348-355.

37. O'Donnell CP, et al. Leptin prevents respiratory depression in obesity. Am J Respir Crit Care Med. 1999;159(5 Pt1):1477-1484.

38. Leinninger GM, et al. Leptin action via neurotensin neurons controls orexin, the mesolimbic dopamine system and energy balance. Cell Metab. 2011;14(3):313-323.

39. Leshan RL, Greenwald-Yarnell M, Patterson CM, Gonzalez IE, Myers MG. Leptin action through hypothalamic nitric oxide synthase-1-expressing neurons controls energy balance. Nat Med. 2012;18(5):820-823.

40. Patterson CM, et al. Ventral tegmental area neurotensin signaling links the lateral hypothalamus to locomotor activity and striatal dopamine efflux in male mice. Endocrinology. 2015;156(5):1692-1700. 